\title{
Robot-assisted Endoscopic Surgery: Removal of a Huge Tricuspid Valve Myxoma
}

\author{
Lun Wu Hung ${ }^{1}$, Cheng Ya Lee ${ }^{1}$, and Bor-Chih Cheng ${ }^{1}$ \\ ${ }^{1}$ Chi Mei Medical Center
}

October 22, 2021

\begin{abstract}
Cardiac myxoma is the most common benign cardiac tumor. Its tremendous size and fragile character severely bother the surgeons. Several minimal invasive approaches had been applied for radical tumor excision. The wound was forcibly enlarged for en-bloc specimen removal and prevention of debris sputtering. We reported a case of huge tricuspid valve (TV) myxoma managed by robot-assisted endoscopic tumor resection and TV repair. The tumor was downsized with a morcellator and removed through a keyhole wound $(1.1 \mathrm{~cm}$ in diameter $)$. The patient recovered uneventfully and was discharged after four days.
\end{abstract}

\section{Introduction}

Myxoma is the most common benign cardiac tumor. The incidence was reported to be $60-80 \%$ at the left atrium, $15-28 \%$ at the right atrium, $8 \%$ at the right ventricle, and $3-4 \%$ at the left ventricle. The atrioventricular valve area was the least common origin $(1 \%)[1,2]$. There are several approaches to remove myxoma, including traditional sternotomy, minimally invasive thoracotomy, and robot-assisted surgery. Robot-assisted endoscopic operation was done for huge tumor excision and tricuspid valve (TV) repair. During the operation, the myxoma was downsized with a morcellator (NOUVAG AG, Inc, Goldach, Switzerland) in the pericardial cavity. Simultaneous TV repair was completed from the endoscopic working ports.

\section{Case Report}

A 56-year-old woman, without previous systemic disease, visited our out-patient department for worsening shortness of breath for two months. A grade II/VI systolic murmur was noted over the 2nd left parasternal border. Jugular vein engorgement was also noted. Transthoracic echocardiogram showed a huge mobile tumor in the right ventricle, originating from the TV and extending into the pulmonary trunk. The tumor stalk, about $12 \mathrm{~mm}$ in diameter, was connected to the anterior tricuspid leaflet and its chordae tendineae. The tumor occupied the right ventricular outflow tract and nearly obstructed the pulmonary valve. The estimated tumor volume based on computed tomography (CT) scan was $69.36 \mathrm{~cm}^{3}$, with a maximum diameter of 41 $\mathrm{mm}$ (Figure 1). An urgent operation was conducted for unstable hemodynamic status.

The operation was performed using a totally endoscopic, robot-assisted approach. After the induction of general anesthesia, the patient was intubated smoothly. A central venous catheter and 6.5-Fr introducer sheath were placed into the right internal jugular vein. A 2-cm oblique right groin incision was made for peripheral cannulation. After systemic heparinization, the patient's femoral artery was cannulated (19 Fr) for systemic retrograde perfusion. Adjunctive distal femoral perfusion was done using an 8-Fr arterial cannula (Medtronic, Minneapolis, MN). Bicaval venous drainage was initiated through the right internal jugular vein (17 Fr) and femoral vein (23 Fr). Cardiopulmonary bypass was established. A camera port $(7 \mathrm{~mm})$ was introduced into the fourth intercostal space (ICS) at the right midclavicular line. The left and right robot arm ports $(7 \mathrm{~mm})$ were placed in the second ICS at the midclavicular line and sixth ICS at the anterior axillary line. The assisting port $(7 \mathrm{~mm})$ was placed in the fourth ICS at the right parasternal border. 
The Da Vinci Xi surgical system (Intuitive Surgical, Inc, Sunnyvale, CA) cart was docked. A 11.5-mm endoscopic trocar port (Thoracoport ${ }^{\mathrm{TM}}$, Medtronic, Minneapolis, MN) was placed at the right fourth ICS. A transthoracic Chitwood cross-clamp (Scanlan International, Minneapolis, MN) was placed at the right fourth ICS at the mid-axillary line. A cardioplegia delivery pig-tail catheter was inserted through the second ICS at the right parasternal border directly into the ascending aorta. Right pericardiotomy was done. After aortic cross-clamping, myocardial protection was achieved using antegrade cold crystalloid cardioplegia with histidine-tryptophan-ketoglutarate solution (30 cc/kg; Custodiol HTK; Köhler Chemie GmbH, Bensheim, Germany). The heart was decompressed by bi-caval snare. Right atriotomy was done. Atrial retractor was applied through the assisting port, and the TV was exposed.

When exploring the tumor, the anterior leaflet of the TV was incised perpendicularly in the midline. The tumor stalk, about $12 \mathrm{~mm}$ in diameter, connects the ventricular side of the anterior leaflet and associated papillary muscle. Hence, the tumor was excised en-bloc with the associated leaflet and papillary muscle tip. It was placed in a wired endo bag (UNIMAX, Taipei, Taiwan (R.O.C.)) inserted from the working port. Then, the tumor was cut into pieces by the morcellator and sucked out without debris sputtering (Figure 2)(Supplemental material).

The anterior leaflet was repaired with CV-5 sutures. One CV-5 artificial cord was applied between the residual papillary muscle and corresponding leaflet edge. A 28-mm annuloplasty ring (MC3, Edwards Lifescience, Irvine, CA) was applied with 10 sets of Cor-knots (LSI SOLUTIONS, Victor, NY) fixation (Figure 3). Normal sinus rhythm resumed after aortic clamp off. The patient weaned off bypass without inotropes. The aortic clamping time was $121 \mathrm{~min}$, and the total cardiopulmonary bypass time was $175 \mathrm{~min}$.

Extubation was done two hours after the surgery. The patient was transferred to the general ward on the next day and was discharged uneventfully on the fourth postoperative day.

\section{Discussion:}

TV tumor is a rare disease. In a collective review, only 51 cases of myxoma originating from the TV were reported by 2008. Most tumors that originated from the TV required TV repair or replacement and myxoma resection (replacement, 22\%; repair, 56.1\%; tumor resection only, 22\%) [3, 4]. The tumor size varies from 1 $\mathrm{cm}$ to $15 \mathrm{~cm}$ in diameter. The larger tumor also appears to be a major risk factor for embolism [5].

In the past two decades, minimally invasive techniques were applied in all areas of cardiac surgery due to admirable cosmetics and speedy postoperative recovery. [6] The Da Vinci robot-assisted cardiac surgery provides stable scope, enhanced visualization by ten-fold, and dexterous surgical arms. Nevertheless, the chest wounds must be widened in extra-large tumor excision despite the minimal operations designed.

The morcellator was initially approved for laparoscopic uterine myoma removal. However, the device was less used recently as per FDA recommendation due to potential malignant tissue spreading. The FDA recommends performing laparoscopic power morcellation for myomectomy only within a well-protected containment system.

In this case, the huge myxoma was excised en-bloc and placed into the endo bag within the pericardial cavity. A morcellator shaft (12 $\mathrm{mm}$ in diameter) was inserted into the endo bag with an attached negative pressure filter system. The downsized tumor strips were removed smoothly with confined debris. In only 5 min, the procedure was completed through the tiny working port.

To our knowledge, there were no previous reports on morcellator-assisted cardiac tumor downsizing and port removal. With the first morcellator application, this might be the smallest surgical wound reported after the removal of a huge cardiac myxoma. With the Da Vinci system, the ICU and hospital stays were shortened. The most concerned cosmetic problems and wound pain were also alleviated. This might be effectively applied to further minimally invasive surgeries for cardiac tumor excision.

Conflict of Interest: There is no acknowledgment, and the authors declare no conflict of interest relevant to the manuscript. 


\section{Author contributions:}

Lun-Wu Hung, M.D: Drafting article, revision of article, data collection and interpretation.

Cheng-Ya Lee, M.D: Drafting article and data collection.

Bor-Chih Cheng, M.D: Designing of the work, critical revision of article, final approval of the version to be published.

\section{Reference:}

1. Igor Gošev, Frane Paić, Željko Đurić et al. Cardiac myxoma the great imitators: Comprehensive histopathological and molecular approach.Int J Cardiol . 2013;164(1):7-20.

2. Boutayeb A, Mahfoudi L and Moughil S. Atrial Myxoma: From Diagnosis to Management. Clin Surg. 2017;2:1498.

3. Shi-Min Yuan, Amihay Shinfeld, Ehud Raanani. Tricuspid valve myxoma: a case report and a collective review of the literature. J Card Surg. 2009;24(1):69-72.

4. B el Asmar, M Acker, J P Couetil, et al. Tricuspid valve myxoma: a rare indication for tricuspid valve repair. Ann Thorac Surg.1991 Dec;52(6):1315-6.

5. Jun Hirota, Kazuya Akiyama, Naohito Taniyasu, et al. Injury to the tricuspid valve and membranous atrioventricular septum caused by huge calcified right ventricular myxoma: report of a case. Circ J.2004 Aug;68(8):799-801.

6. Cristina Barbero, Giovanni Marchetto, Davide Ricci, et al. Steps Forward in Minimally Invasive Cardiac Surgery: 10-Year Experience.Ann Thorac Surg. 2019 Dec;108(6):1822-1829.

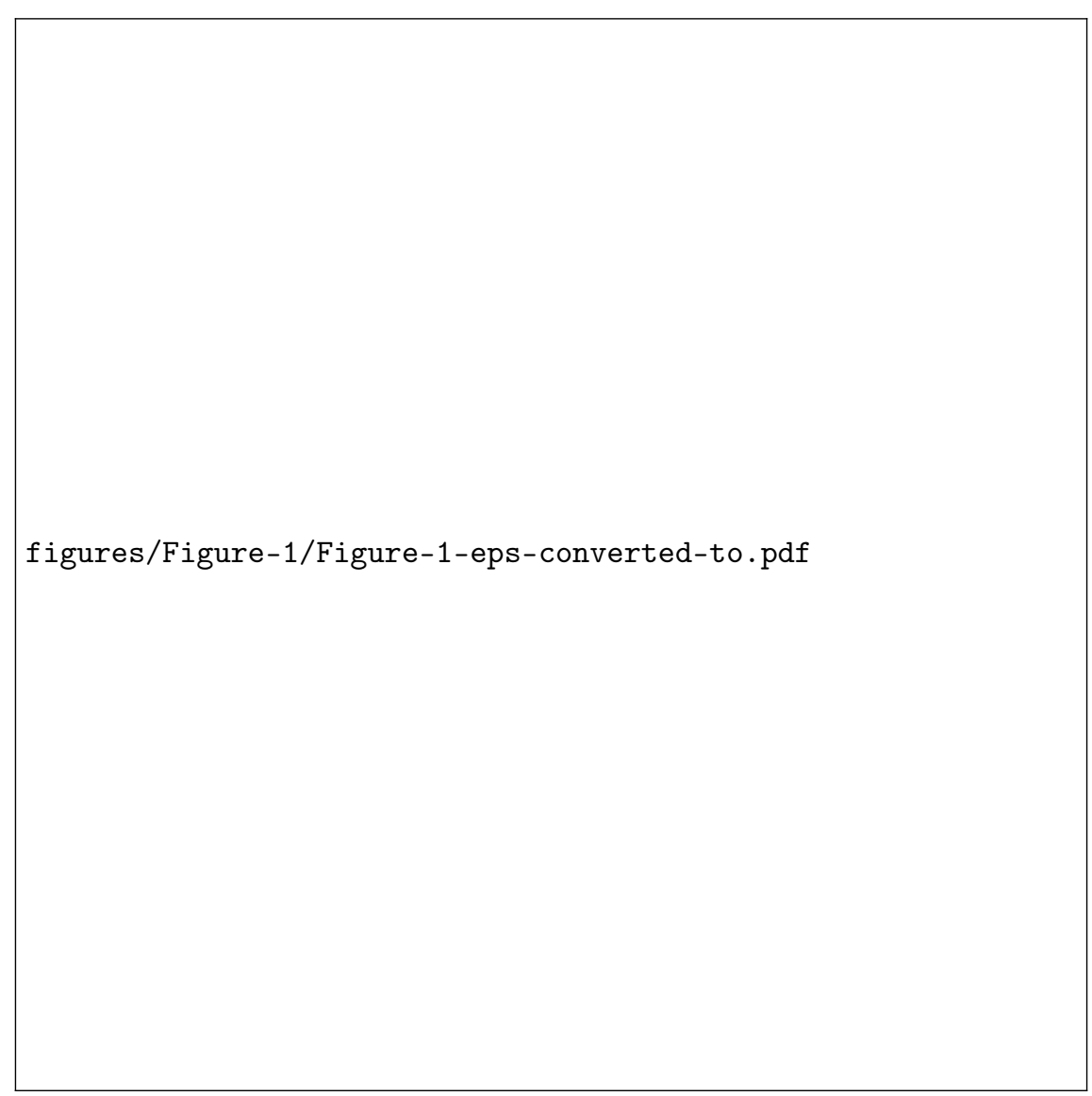



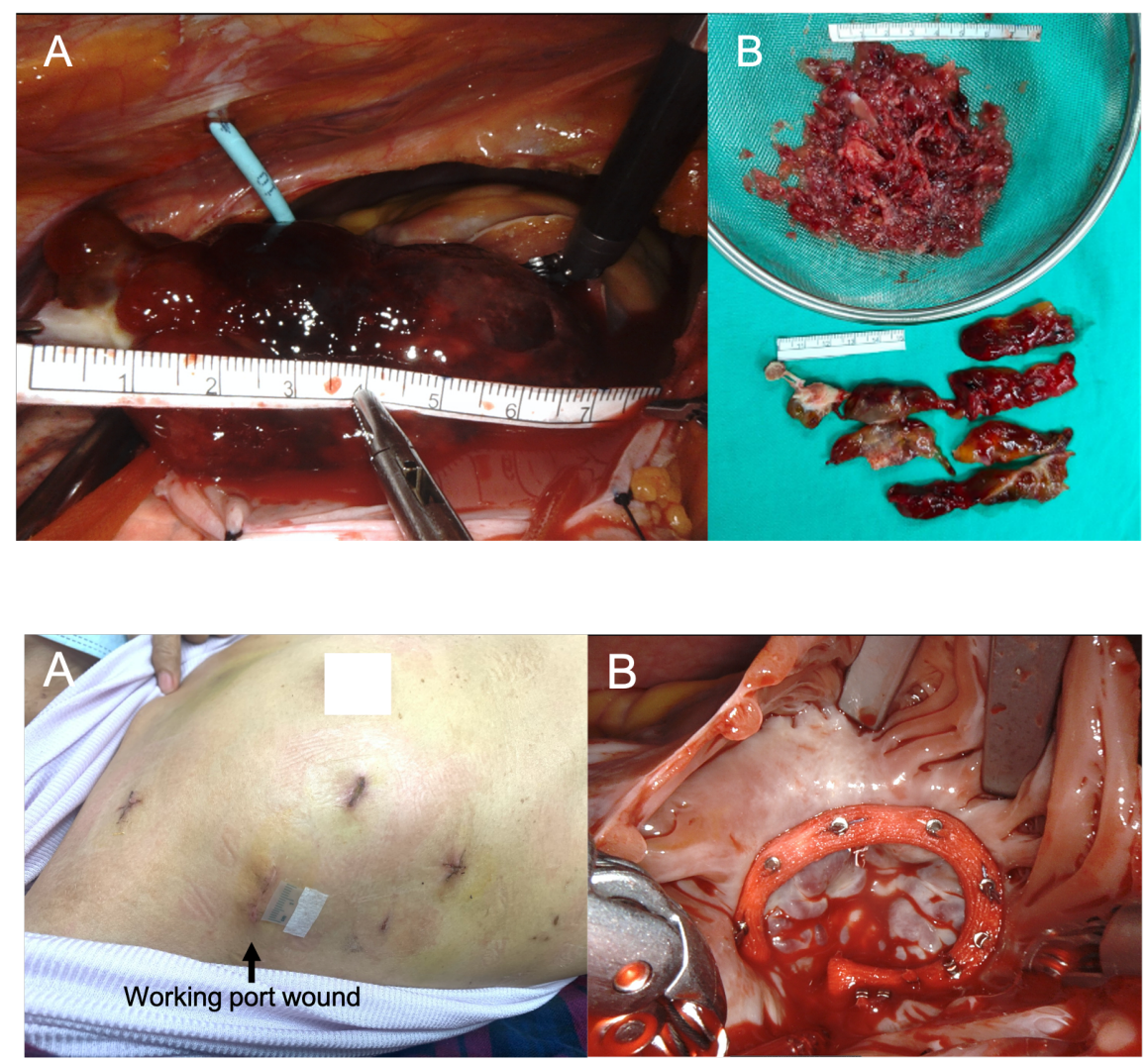